\title{
Tiered Licensed-Assisted Access with Paid Prioritization: A Game Theoretic Approach for Unlicensed LTE
}

\author{
Ting-Hsuan $\mathrm{Wu}^{*}$, Mei-Ju Shih ${ }^{\dagger}$, and Hung-Yu Wei*†† \\ *Department of Electrical Engineering, National Taiwan University, Taiwan \\ ${ }^{\dagger}$ Graduate Institute of Communication Engineering, National Taiwan University, Taiwan \\ ${ }^{\ddagger}$ Corresponding Author: hywei@cc.ee.ntu.edu.tw
}

\begin{abstract}
The network congestion is caused by the rapidly growing data traffic and the limited wireless radio resources. In addition to the licensed spectrum, the access to unlicensed spectrum (e.g., LAA) brings hope for the service provider (SP) to mitigate the deficiency of radio resources. The premium peering deal with the content providers (CPs) can be an approach to efficiently allocate the scarce radio resources to the CPs with higher traffic load and QoS requirement. This work contributes to a content premium pricing framework for one SP and several CPs, where the SP possesses both LTE and LAA. Through the four-stage Stackelberg game, job market signaling game and second price auction, we derive the optimal bandwidth demand of each CP, the optimal amounts of licensed bandwidth and unlicensed bandwidth required by the $S P$, the premium access fee and basic access fee. Analysis shows that the CPs and the SP all benefit from the premium access deal. Furthermore, there is a tradeoff between improvement and variability of the SP's profit when introducing LAA.
\end{abstract}

\section{INTRODUCTION}

As the network traffic is expected to rise 1000 times in ten year [1], traffic congestion management has been a challenging issue for service providers (SPs) under the constraint of scarce wireless resources. Traditionally, network pricing has been a good approach to manage the traffic congestion. For example, SPs charge end users (EUs) for the broadband access to the network so as to alleviate the congestion level. Recently, peering agreements among content providers (CPs) and SPs have become popular with the purpose of granting higher speed resources for CPs with enormous traffic requirement. Thus, the two-sided market forms, where EUs and CPs are the two sides of SPs. In addition to the licensed spectrum, unlicensed spectrum access technology is considered by SPs as a solution to accommodate more users. In this paper, considering the scenario where one SP adopts both licensed and unlicensed wireless broadband access technologies (e.g., LTE and LAA), we propose the network pricing models with premium peering among CPs and the SP.

The $3^{r d}$ Partnership Project (3GPP) has kicked off a study item on a single global framework for licensed-assisted access (LAA) to unlicensed spectrum [2], also called LTE-U. LAA not only leverages SPs' existing investment on LTE equipments, but also caters the significantly increasing demand for wireless broadband data. Inter-technology coexistence mechanisms, e.g., reusing the blank subframe approach and the uplink power control management proposed in [3], are required to improve the fairness, interference and performance of both LAA and existing unlicensed radio access technology such as IEEE 802.11. The concept of ISM-Advanced in [4] considers to incorporate cognitive radio capabilities into the rule for unlicensed spectrum access in ISM bands (Industrial Scientific Medical Band) so as to improve the spectrum efficiency and QoS (Quality of Service). Through spectrum sensing, a cognitive mobile virtual network operator (C-MVNO) can improve its expected profit and users' payoff by considering the cost and uncertainty of the spectrum [5]. From a service provider's perspective, the pricing model can turn over a new leaf in the co-existence of existing wireless broadband network with leased bandwidth (e.g. LTE) and promising radio access technology with unlicensed bandwidth (e.g. LAA).

Premium peering agreement appears in the network market among CPs and the SP, where CPs with higher traffic requirements pay more so that it can gain extra resource access and be guaranteed with higher QoS to satisfy their subscribers. For example, Netflix signed a deal with AT\&T in July 2014 to deliver its streaming content via wireless broadband network with higher capacity. The design of AT\&T sponsored data program in [6] is developed via a Stackelberg game between an SP leader and a CP follower. A utility maximization based rate allocation model with pricing CPs in [7] indicates that the CPs can gain net surplus in the monopolistic and competitive market. An analysis for premium peering using real world data is carried out in [8], but the proposed Nash bargaining pricing scheme can be infeasible because the SP grants the premium access for free.

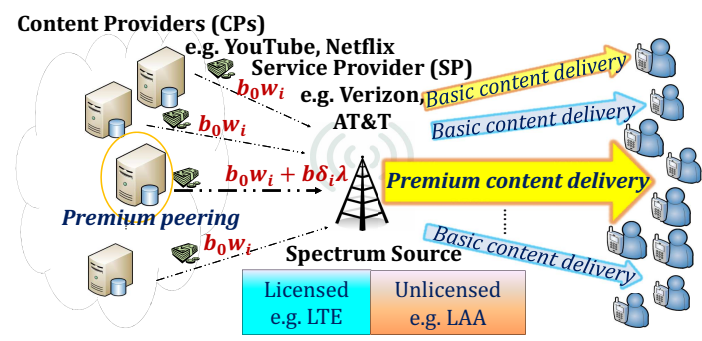

Fig. 1. System model

This work contributes a premium peering pricing model considering the SP provides both LTE and LAA wireless 
broadband access technologies. The SP, as a Stackelberg leader, decides the amount of licensed and unlicensed bandwidth. Through job market signaling game and second price auction, the premium access price is determined among the bids offered by several CPs. Those CPs, as the Stackelberg followers, thereby determine their bandwidth demand. This research brings insights on the benefit of premium peering deal and LAA to confront the soaring traffic requirement. The rest of this paper is organized as follows. In Section II, we describe the system model with one SP and several CPs. The demand and supply for the bandwidth, the premium access price and the basic access fee are derived via backward induction in Section III. Numerical results are presented in Section IV. Section V concludes this work.

\section{SYSTEM MODEL}

We consider one SP, which operates a wireless broadband Internet network and $M$ CPs participate to deliver their content, as illustrated in Fig. 1. To satisfy the increasing wireless resource demand from the CPs in each time slot, the SP further introduces LAA on the unlicensed band in addition to the existing LTE on the licensed band. The amount of licensed bandwidth is denoted as $B_{l} \in\left[0, B_{l}^{\max }\right]$, and that of the unlicensed bandwidth is denoted as $B_{u} \in\left[0, B_{u}^{\max }\right]$. However, several wireless technologies such as $\mathrm{WiFi}$ and bluetooth operating on the shared unlicensed band can cause interference and degrade the performance of LAA. Therefore, we model the unreliability property of LAA with $\alpha \in[0,1]$, the access reliability factor. The realized effective bandwidth of LAA can be $\alpha B_{u}$. The SP first determines the amount of unlicensed bandwidth $B_{u}$, and then the amount of licensed bandwidth $B_{l}$. The total available bandwidth for the SP to deliver the content from CPs is $B_{l}+\alpha B_{u}$.

After obtaining spectrum for LTE and LAA, the SP announces the power allocation factor $\lambda$ and negotiates the issues of premium peering with CPs. The choice of $\lambda$ is made by the SP beforehand and detailed in stage III. As equipment constraints are common for SPs, we assume that the SP executes power allocation for one specific $\mathrm{CP}$, called $\mathrm{CPH}$, to allow its premium access by delivering its content with higher data rate. For each $\mathrm{CP} i$, the aggregate content delivery rate (in nats) is

$$
r_{i}\left(w_{i}\right)=w_{i} \ln \left(1+\frac{P^{\max } h_{i}\left(1+\delta_{i} \lambda\right)}{n_{0} w_{i}}\right),
$$

where $w_{i}$ is the allocated bandwidth, $P^{\max }$ is the SP's maximum transmission power, $n_{0}$ is the noise spectral density, $h_{i}$ is the channel gain among the SP and CP $i$ 's subscribers. $\lambda$ is power allocation factor for CP $i$, which improves CP $i$ 's $\mathrm{SNR}$. We assume that only the $\mathrm{CPH}$ is provided with the power allocation for SNR improvement. Therefore, $\delta_{i}=1$ when CP $i$ is the CPH. Otherwise, $\delta_{i}=0$ without premium access. In addition to the identical price $b_{0}$ per unit bandwidth charged to all the CPs, CPH pays extra fee $b \lambda$ for its premium access service. That is, the total price charged to the $\mathrm{CPH}$ includes the basic service $b_{0} w_{i}$ and premium access $b \lambda$.

In a paid peering deal, power allocation technique is a critical element for it distinguishes the $\mathrm{CPH}$ from the rest of the CPs. As literature suggests, operating ability generates profits through utilizing resources efficiently. High-ability SPs often receive loans with favorable terms [9], and capable to upgrade equipments to improve the CPH's SNR. Operating ability cannot be directly observed by CPs, but CPs can assess the true operating ability through observable information, e.g., level of certification, financial records, news reports, etc. Despite the fact that some observable information is not at the discretion of the SP, the level of certification is what can be invested with time and money [10]. Thus, we refer to the level of certification as a signal emitted by the SP to determine the paid peering deal. After the SP announces the basic price $b_{0}$ and premium access price $b$, each $\mathrm{CP}$ ask the SP for its required bandwidth to maximize its payoff.

$$
u_{i}\left(b_{0}, b, w_{i}\right)=r_{i}\left(w_{i}\right)-b_{0} w_{i}-b \delta_{i} \lambda,
$$

which is the difference between the aggregate data rate and total payment for service.

The system is modelled as a four-stage Stackelberg game as shown in Fig. 2, where the SP is the leader and CPs are the followers. First, the SP decides the amount of unlicensed bandwidth $B_{u}$ for LAA. Then, it determines the amount of licensed bandwidth based on the realized effective unlicensed spectrum. In stage 3, the SP grants premium access through job market signaling game and second price auction, and announces the $\mathrm{CPH}$ and prices, $b_{0}$ and $b$, based on the total effective spectrum supply $\alpha B_{u}+B_{l}$. Finally, CP $i$ chooses its bandwidth demand $w_{i}$ to maximize its payoff. The key notations are listed in Table I.

TABLE I. TABLE OF NOTATIONS

\begin{tabular}{lll}
\hline \hline Symbol & Definition & $($ Setting) \\
\hline$B_{u} \in\left[0, B_{u}^{\text {max }}\right]$ & The amount of unlicensed bandwidth & $\left(B_{u}^{\text {max }}=80 \times 10^{6}\right)$ \\
$B_{l} \in\left[0, B_{l}^{\text {max }}\right]$ & The amount of licensed bandwidth & $\left(B_{u}^{\text {max }}=20 \times 10^{6}\right)$ \\
$M$ & Number of CPs & $\left(M=10^{3}\right)$ \\
$\alpha \in[0,1]$ & Access reliability factor in LAA & \\
$\eta$ & Operating ability, $\eta=\{h i g h$, low $\}$ & $(\lambda=0.5)$ \\
$\lambda$ & Power allocation factor in CP $i$ & $\left(\mu=10^{4}\right)$ \\
$\mu$ & Cost of improved power allocation & \\
$b_{0}$ & Price per unit bandwidth & \\
$b \delta_{i}$ & Additional unit fee for the improvement in & \\
& SNR & $\left(c_{u}=0.8\right)$ \\
$c_{u}$ & Cost per unlicensed bandwidth & $\left(c_{l}=2\right)$ \\
$c_{l}$ & Cost per licensed bandwidth & \\
$s$ & Level of certification & \\
$w_{i}$ & CP $i$ 's bandwidth allocation & \\
\hline \hline
\end{tabular}

Stage 1: The SP determines the unlicensed spectrum $B_{u}$
\begin{tabular}{|l|}
\hline Stage 2: \\
Stage 3: The SP determines the licensed spectrum $B_{l}$
\end{tabular}
Stage 4: CPs determine the demands for bandwidth

Fig. 2. Stackelberg game

\section{BACKWARD INDUCTION: JOB MARKET SIGNALING GAME APPROACH}

Stackelberg game falls in the category of the dynamic game, whose solution concept is the Subgame perfect equilibrium (SPE). The SPE is often determined through backward induction by examining that each subgame reaches the Nash 
equilibrium. Hence, the four-stage Stackelberg game can be solved sequentially via backward induction to achieve the SPE.

\section{A. Stage 4 : CPs' Bandwidth Demand}

After acquiring the knowledge of the premium access price $b$ and the basic price $b_{0}$ from the SP, CP $i$ determines its bandwidth requirement $w_{i}$ to deliver its content. From $\mathrm{CP}$ $i$ 's point of view, it can not distinguish whether its content is delivered via LTE or LAA. We assume CP $i$ knows the SP's maximum transmission power $P^{\max }$ and the channel gain between SP to its subscribers $h_{i} . h_{i}$ and $w_{i}$ are private information to $\mathrm{CP} i$ itself.

To simplify the notations, let $g_{i}=P^{\max } h_{i} / n_{0}$. The SNR of CP $i$ 's aggregate delivery can be written as $g_{i}\left(1+\delta_{i} \lambda\right) / w_{i}$. To obtain closed-form solutions, we focus on the high SNR regime where $S N R \gg 1$ with good signal quality to deliver the content. Thus, CP $i$ 's aggregate content delivery rate in Eq. (1) can be approximated as $r_{i}\left(w_{i}\right)=w_{i} \ln \left(\frac{g_{i}\left(1+\delta_{i} \lambda\right)}{w_{i}}\right)$, and its payoff is

$$
u_{i}\left(b_{0}, b, w_{i}\right)=w_{i} \ln \left(\frac{g_{i}\left(1+\delta_{i} \lambda\right)}{w_{i}}\right)-b_{0} w_{i}-b \delta_{i} \lambda .
$$

Here we implicitly assume that content delivery rate can be transformed to some forms of monetary benefits, a common assumption in game theory literature. Because $u_{i}\left(b_{0}, b, w_{i}\right)$ is concave in $w_{i}$, the unique bandwidth demand $w_{i}$ can be obtained in Eq. (2) via differential operation to maximizes CP $i$ 's payoff.

$$
w_{i}^{*}\left(b_{0}, b\right)=\underset{w_{i} \geq 0}{\arg \max } u_{i}\left(b_{0}, b, w_{i}\right)=g_{i}(1+\lambda) e^{-\left(1+b_{0}\right)}
$$

Eq. (2) is always positive, linear in $g_{i}$, and decreasing in price $b_{0}$. Since $g_{i}$ is linear in channel gain $h_{i}$ and transmission power $P^{\max }$, a better channel condition or a larger transmission power will increase CP $i$ 's demand. It is clear that $w_{i}{ }^{*}\left(b_{0}, b\right)$ is upper bounded by $g_{i}\left(1+\delta_{i} \lambda\right) e^{-1}$ for any choice of price $b_{0} \geq 0$. In other words, even if the SP announces a zero price $b_{0}=0, \mathrm{CP} i$ will not purchase infinite amount of radio resources. The reason is that infinite bandwidth demand leads to the reduced received SNR, and the subscribers cannot detect the signal carrying the delivered content when the received SNR is below a threshold.

We also consider the situation that premium peering is forbidden (i.e., $b=0$ and $\lambda=0 \forall i$ ). Derive from Eq. (2), all CPs achieve the same SNR in Eq. (3), but different payoffs in Eq. (4) which are linear in $g_{i}$.

$$
\begin{gathered}
S N R_{i}=\frac{g_{i}}{w_{i}^{*}\left(b_{0}\right)}=e^{1+b_{0}} \\
u_{i}\left(b_{0}, w_{i}^{*}\left(b_{0}\right)\right)=g_{i} e^{-\left(1+b_{0}\right)}
\end{gathered}
$$

Next, we consider the total bandwidth demand of all CPs. The bandwidth demand function $D$ is

$$
D\left(b_{0}, b\right)=\sum_{i \in M} w_{i}^{*}\left(b_{0}, b\right)=G e^{-\left(1+b_{0}\right)},
$$

where $G=\sum_{i \in M} g_{i}\left(1+\delta_{i} \lambda\right)$. The demand $D$ is the same as the total bandwidth supply by setting $b_{0}$ equal to the market clearing price. Usually, the licensed and unlicensed bandwidth is scarce in nature. Thus, the demand which is served by the $\mathrm{SP}$ is referred to as the realized demand $Q$, which is the total effective bandwidth supply in Eq. (6).

$$
Q\left(\alpha, B_{u}, B_{l}, b_{0}, b\right)=\alpha B_{u}+B_{l}
$$

Because the power allocation rule and prices announced by the SP have a great impact on the Stackelberg followers' bandwidth demand, the realized demand $Q$ can be learned by the Stackelberg leader in advance.

\section{B. Stage 3 : Optimal Pricing}

The SP resolves the optimal pricing, i.e., the additional premium access fee $b^{*} \lambda$ and the basic fee $b_{0}^{*}$, considering the realized demand $Q$ in Eq. (6) where the total supply $B_{u} \alpha+$ $B_{l}$ is obtained in stage 2 . The SP's profit from providing the licensed and unlicensed spectrum to CPs is

$$
\begin{aligned}
\pi^{C P}\left(\alpha, B_{u}, B_{l}, b_{0}, b\right)= & b_{0} Q\left(\alpha, B_{u}, B_{l}, b_{0}, b\right)+b \lambda \\
& -B_{u} c_{u}-B_{l} c_{l}-\mu \lambda
\end{aligned}
$$

which is the difference between its revenue from the CPs and its total cost. The cost comes from the investment on the licensed LTE, unlicensed LAA and power allocation equipments. $\mu$ can be viewed as the cost of improved SNR claimed by the SP. $c_{l}$ and $c_{u}$ are the cost of unit bandwidth for licensed LTE and unlicensed LAA, respectively.

Since the payment $B_{u} c_{u}+B_{l} c_{l}+\mu \lambda$ is fixed at this stage, the SP's profit maximization problem is the equivalent of maximization of its revenue $b_{0} Q+b \lambda$. The objective of stage 3 is to find the optimal prices $b_{0}^{*}$ and $b^{*}$ that maximize the profit from the spectrum allocation, i.e.,

$$
\pi_{3}^{C P}\left(\alpha, B_{u}, B_{l}\right)=\max _{b_{0}, b>0} \pi_{4}^{C P}\left(\alpha, B_{u}, B_{l}, b_{0}, b\right)
$$

The subscript "3" and "4" denotes the best profit in stage 3 and 4.

The solution of Eq. (7) depends on the spectrum investment in stage 1 and 2 . When the total supply exceeds $G e^{-2}, Q=$ $\sum_{i \in M} w_{i}{ }^{*}\left(b_{0}, b\right)$. The solution of Eq. (7) occurs at the maximum value of Eq. (5) with $b_{0}^{*}=1$. On the other hand, when the total supply is below $G e^{-2}, Q=\alpha B_{u}+B_{l}$. In this case, $b_{0}$ is the market clearing price which sets Eq. (5) equal to $\alpha B_{u}+B_{l}$. The optimal $b_{0}^{*}$ and the corresponding optimal profit are summarized by Table II. The derivation of Table II was basically mentioned in [5], but here we also take into account the profit which arises from premium peering.

Eq. (7) indicates that the total supply of spectrum exceeds $G e^{-2}$ happens when spectrum reserve is much greater than spectrum demanded, and contradicts the fact that spectrum scarcity is a prevalent problem today. In addition, if the acquired bandwidth is too large, selling all the bandwidth will lead to a very low price that decreases the revenue. The profit can be apparently improved if the SP acquires less bandwidth in stages 1 and 2 . Therefore, we will focus on the case where the total supply does not exceed $G e^{-2}$ in the rest of this paper.

Another issue is to determine the $\mathrm{CPH}$ and the additional fee $b \lambda$ it contributes. Each CP offers its bid for the expected premium access based on the signal sent by the SP. We then combine job market signaling game with second price auction. 
TABLE II. PRICING DeCision ANd PRofit IN Stage 3

\begin{tabular}{|l|l|l|}
\hline Total Bandwidth & Optimal Price $b_{0}^{*}\left(B_{u}, \alpha, B_{l}\right)$ & Optimal Profit $\pi_{3}^{C P}\left(B_{u}, \alpha, B_{l}\right)$ \\
\hline$B_{u} \alpha+B_{l} \geq G e^{-2}$ & $b_{0}=1$ & $\pi_{3}^{C P}\left(B_{u}, \alpha, B_{l}\right)=G e^{-2}+b \lambda-B_{u} c_{u}-B_{l} c_{l}-\mu \lambda$ \\
\hline$B_{u} \alpha+B_{l}<G e^{-2}$ & $b_{0}=\ln \left(\frac{G}{B_{u} \alpha+B_{l}}\right)-1$ & $\pi_{3}^{C P}\left(B_{u}, \alpha, B_{l}\right)=\left(B_{u} \alpha+B_{l}\right) \ln \left(\frac{G}{B_{u} \alpha+B_{l}}\right)+b \lambda-B_{u}\left(\alpha+c_{u}\right)-B_{l}\left(\alpha+c_{l}\right)-\mu \lambda$ \\
\hline
\end{tabular}

A second price auction is a type of sealed-bid auction in which the highest bid wins but the paid price is the second-highest bid; studies show that it insures the property of truth-telling [11]. The mechanism is presented as follows:

1) Nature determines the SP's operating ability, $\eta$, which can be either high $(h)$ or low $(l)$. The probability of which $\eta=h$ is $q$.

2) The SP learns its ability and then chooses a level of certification related to maintaining high speed, $s \geq 0$. (e.g., Internet speed survey, ISO/IEC 27001, etc.)

3) $\quad M$ CPs observe the SP's certification and then simultaneously make price offers to the SP.

4) The SP accepts the highest bid among the $M$ price offers (i.e., $b_{i}, i=1,2,3, \ldots, M$ ), flipping a coin in case of a tie. Let $b^{*}$ denotes the second highest price the SP receives.

Regarding the price of the additional access fee $b_{i}$, Proposition 1 presents its feasible range.

Proposition 1. The range of the optimal additional fee $b^{*}$ is given by

$$
\mu_{*}<b^{*} \leq \frac{w_{i} \ln \left(g_{i}(1+\lambda) / w_{i}\right)-b_{0} w_{i}}{\lambda}
$$

Proof 1. Competition among CPs will drive the expected utility to zero; thus, each $\mathrm{CP} k$ makes bid $b_{k}$ so that its utility $u_{k}=0$ explained in Eq. (1).

$$
u_{k}=w_{k} \ln \left(g_{k}(1+\lambda) / w_{k}\right)-b_{0} w_{k}-b_{k} \lambda=0
$$

Assume $\mathrm{CP} i$ is the selected CPH because its bid is the highest, $b_{i} \geq b_{k}, i \neq k$. The second higher bid is denoted as $b^{*}$ where $b_{i} \geq b^{*}$. Therefore, the utility of CP $i$ will be greater or equal to 0 as shown in Eq. (10).

$$
u_{i}=w_{i} \ln \left(g_{i}(1+\lambda) / w_{i}\right)-b_{0} w_{i}-b^{*} \lambda \geq 0
$$

However, the SP may not maintain the power allocation system with prudence after signing the peering deal. To avoid such principal-agent problem [12], an incentive in the contract to the SP should consider $b^{*} \lambda-\mu_{*} \lambda>0$, which implies Eq. (11).

$$
b^{*} \lambda-\mu_{*} \lambda>0
$$

where $\mu_{*}$ is the optimal lower bound derived from optimal auction theory [13]. Since any $\mathrm{CP} k$ could be the $\mathrm{CPH}$, by combining Eqs. (10) and (11), we have $\mu \lambda<b^{*} \lambda \leq w_{i} \ln \left(g_{i}(1+\right.$ $\left.\lambda) / w_{i}\right)-b_{0} w_{i}$.

Every $\mathrm{CP}$ also bids its true value for the premium peering access, as presented in Proposition 2.

Proposition 2. The bidding process of the additional unit fee $b$ insures the property of truth-telling. $C P i$ bids with $b_{i}$ to reveal its truthful valuation on the premium access.

Proof 2. Define bidder $i$ 's true value for premium access as $v_{i}=w_{i} \ln \left(\left(g_{i} \lambda\right) / w_{i}\right)$; thus the utility for bidder $i$ is

$$
\left\{\begin{array}{cl}
{\left[w_{i} \ln \left(g_{i} / w_{i}\right)+v_{i}\right]-b_{0} w_{i}} & \\
-\left(\max _{k \neq i} b_{k}\right) \lambda, & \text { if } b_{i}>\max _{k \neq i} b_{k} \\
w_{i} \ln \left(g_{i} / w_{i}\right)-b_{0} w_{i}, & \text { otherwise }
\end{array}\right.
$$

The strategy of overbidding is dominated by bidding truthfully. Assume that $b_{i} \lambda>v_{i}>\mu \lambda$.

- If $\max _{k \neq i} b_{k} \lambda<v_{i}$, then the bidder would win the bid with a truthful bid as well as an overbid. The bid price does not change the utility so the two strategies have equal utilities in this case.

- If $\max _{k \neq i} b_{k} \lambda>b_{i} \lambda$, then the bidder would lose the bid either way so both strategies have equal utilities.

- If $v_{i}<\max _{k \neq i} b_{k} \lambda<b_{i} \lambda$, then only the strategy of overbidding would win the auction. The utility would be negative for the strategy of overbidding because it paid more than its value of the bid, while the utility for a truthful bid would be zero.

Thus the strategy of bidding higher than one's true valuation is dominated by the strategy of truthfully bidding.

The strategy of underbidding is dominated by bidding truthfully. Assume that $\mu \lambda<b_{i} \lambda<v_{i}$.

- If $\max _{k \neq i} b_{k} \lambda>v_{i}$, then the bidder would lose the bid with a truthful bid as well as an underbid, so the strategies have equal utilities for this case.

- If $\max _{k \neq i} b_{k} \lambda<b_{i} \lambda$, then the bidder would win the bid either way so both strategies have equal utilities.

- If $b_{i} \lambda<\max _{k \neq i} b_{k} \lambda<v_{i}$, then only the strategy of truthfully bidding would win the auction. The utility for the truthful strategy would be positive as it paid less than its value of the bid, while the utility for an underbid bid is strictly inferior to the former.

Thus the strategy of underbidding is dominated by the strategy of truthfully bidding. Truthful bidding dominates the other possible strategies (underbidding and overbidding) so it is an optimal strategy.

\section{Stage 2 : Optimal Amount of Licensed Spectrum}

The SP decides the optimal licensed amount $B_{l}$ in LTE given the unlicensed amount $B_{u}, B_{l}^{\max }=G e^{-2}-B_{u} \alpha$.

$$
\pi_{2}^{C P}\left(\alpha, B_{u}\right)=\max _{0 \leq B_{l} \leq G e^{-2}-B_{u} \alpha} \pi_{3}^{C P}\left(\alpha, B_{u}, B_{l}\right)
$$

To solve Eq. (12), we need to consider the bandwidth obtained from unlicensed spectrum. By taking the partial derivatives of $\pi_{2}^{C P}\left(\alpha, B_{u}\right)$ with respect to $B_{l}$, we obtain the optimal licensed amount and the optimal profit, summarized in Table III.

From Table III, it is clear that we have an optimal threshold policy here: the SP would like to achieve a total bandwidth equal to $G e^{-\left(2+c_{l}\right)}$ whenever possible. When the bandwidth obtained from unlicensed spectrum is not enough, the SP obtains additional bandwidth from licensed spectrum to reach the threshold, or the SP does not acquire licensed bandwidth. 
TABle III. Optimal Licensed Amount and Profit in Stage 2

\begin{tabular}{|l|l|l|}
\hline Given the unlicensed amount $B_{u} \alpha$ after Stage 1 & Optimal Licensed Amount $B_{l}{ }^{*}$ & Optimal Profit $\pi_{2}^{C P}\left(B_{u}, \alpha\right)$ \\
\hline$B_{u} \alpha \leq G e^{-\left(2+c_{l}\right)}$ & $B_{l}=G e^{-\left(2+c_{l}\right)}-B_{u} \alpha$ & $\pi_{2}^{C P}\left(B_{u}, \alpha\right)=G e^{-\left(2+c_{l}\right)}+B_{u}\left(\alpha c_{l}-c_{u}\right)+b \lambda-\mu \lambda$ \\
\hline$B_{u} \alpha \in\left(G e^{-\left(2+c_{l}\right)}, G e^{-2}\right]$ & $B_{l}=0$ & $\pi_{2}^{C P}\left(B_{u}, \alpha\right)=B_{u} \alpha \ln \left(\frac{G}{B_{u} \alpha}\right)-B_{u}\left(\alpha+c_{u}\right)+b \lambda-\mu \lambda$ \\
\hline
\end{tabular}

TABle IV. Choice of Optimal Unlicensed Amount in Stage 1

\begin{tabular}{|l|l|}
\hline Optimal Unlicensed Amount $B_{u}{ }^{*}$ & Expected Profit from CPs $\pi^{C P}$ \\
\hline Solution to Eq. (14) & $\pi^{C P}$ in Eq. (15) \\
\hline
\end{tabular}

\section{Stage 1 : Optimal Amount of Unlicensed Spectrum}

The SP decides the optimal unlicensed amount to maximize its expected profit by considering the uncertainty of the access reliability factor $\alpha$ in LAA. The SP needs to solve the following problem:

$$
\pi^{C P}=\max _{0 \leq B_{u} \leq B_{u}^{\max }} E\left(\pi_{2}^{C P}\left(\alpha, B_{u}\right)\right),
$$

by taking the expectation of $\alpha$ over the profit functions in stage 2 (i.e., the optimal profit functions in Table III). We assume that the access reliability factor $\alpha$ follows a uniform distribution in $[0,1]$. To avoid the case where unlimited unlicensed bandwidth is optimal, we refer to [5] and further assume that the cost of unlicensed bandwidth lies in $\left[\left(1-e^{-2 c_{l}}\right) / 4, c_{l} / 2\right]$.

The function $E\left(\pi_{2}^{C P}\left(\alpha, B_{u}\right)\right)=\pi_{2}^{C P}\left(B_{u}\right)$ is derived:

$$
\begin{aligned}
& \pi_{2}^{C P}\left(B_{u}\right)=E_{\alpha \in\left[0, \frac{G e^{-\left(2+c_{l}\right)}}{B_{u}}\right]}\left[G e^{-\left(2+c_{l}\right)}+B_{u}\left(\alpha c_{l}-c_{u}\right)+(b-\mu) \lambda\right] \\
& +E_{\alpha \in\left[\frac{G e^{-\left(2+c_{l}\right)}}{B_{u}}, 1\right]}\left[B_{u} \alpha \ln \left(\frac{G}{B_{u} \alpha}\right)-B_{u}\left(\alpha+c_{u}\right)+(b-\mu) \lambda\right] \\
& \left.=\frac{B_{u}}{2} \ln \left(\frac{G}{B_{u}}\right)-\frac{B_{u}}{4}\left(\frac{G e^{-\left(2+c_{l}\right)}}{B_{u}}\right)^{2}-B_{u} c_{u}+(b-\mu) \lambda\right] .
\end{aligned}
$$

$\pi_{2}^{C P}\left(B_{u}\right)$ is a strictly concave function of $B_{u}$ since its second-order derivative

$$
\frac{\partial^{2} \pi_{2}^{C P}\left(B_{u}\right)}{\partial B_{u}{ }^{2}}=\frac{1}{2 B_{u}}\left[\left(\frac{G e^{-\left(2+c_{l}\right)}}{B_{u}}\right)^{2}-1\right]<0 .
$$

Table $\mathrm{V}$ describes briefly the unlicensed amount obtained by the SP. The optimal unlicensed amount $B_{u}{ }^{*}$ is the unique solution to the following equation:

$$
\frac{\partial^{2} \pi_{2}^{C P}\left(B_{u}\right)}{\partial B_{u}}=\frac{1}{2} \ln \left(\frac{1}{B_{u} / G}\right)-\frac{3}{4}-c_{u}-\left(\frac{e^{-\left(2+c_{l}\right)}}{2 B_{u} / G}\right)^{2}=0 .
$$

The uniqueness of the solution is due to the strict concavity of $\pi_{2}^{C P}\left(B_{u}\right)$ over $B_{u} . B_{u}{ }^{*}$ lies in $\left[G e^{-\left(2+c_{l}\right)}, G e^{-2}\right]$ and is linear in $G$. Finally, the SP 's optimal expected profit is

$$
\pi^{C P}=\frac{B_{u}^{*}}{2} \ln \left(\frac{G}{B_{u}^{*}}\right)-\frac{B_{u}^{*}}{4}+\frac{1}{4 B_{u}^{*}}\left(\frac{G}{e^{2+c_{l}}}\right)^{2}-B_{u}^{*} c_{u}+(b-\mu) \lambda .
$$

The optimal unlicensed amount is summed up in Table IV. Table V summarizes the SP's equilibrium spectrum amount, pricing decisions and resource allocation to the $\mathrm{CPH}$.

\section{NUMERICAL ANALYSIS}

Considering the access reliability factor of LAA spectrum, we begin with the comparison between the impact of premium peering (PP) and network neutrality (NN) on the profit of the $\mathrm{SP}$ and CPs. When NN rules, the SP is not allowed to charge any payment from CPs and does not provide the opportunity for premium peering, i.e., $b_{0}=0, b=0$ and $\lambda=0$ for all CPs.

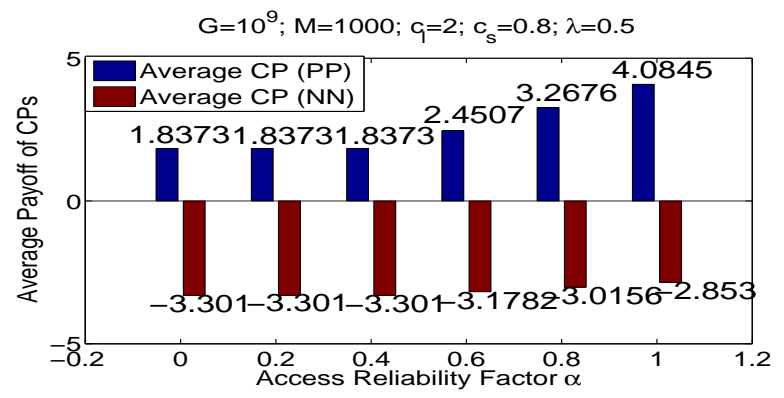

Fig. 3. Effect of Premium Peering on the Payoff of CPs

In Fig. 3, we observe that under NN the average payoff of CPs is lower than that when premium peering exists. We also calculate the coefficient of variance $(\mathrm{CV})$ of resource per $\mathrm{CP}$ : the CV for PP is 1.0585 and that for NN is 2.9464. Under NN, though CPs use the radio resources for free, a lack of price signals results in excess demand for bandwidth: all CPs request a large amount of bandwidth, but some of them attain it and others receive none. Conversely, when PP exists, the supply and demand are aligned and the payoff of CPs on average is always positive.

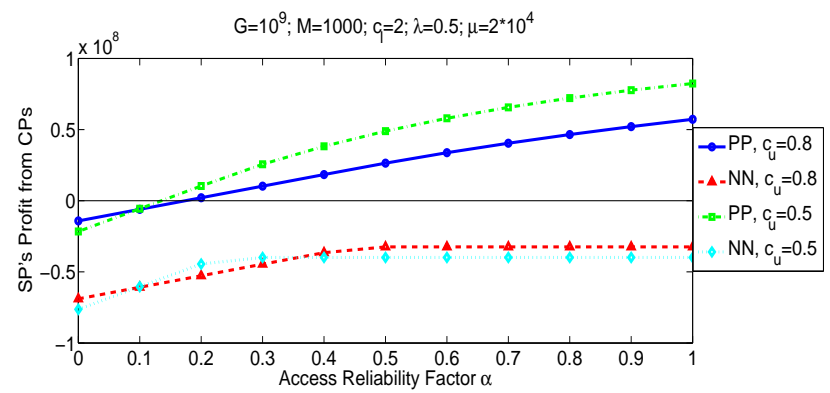

Fig. 4. Effect of Premium Peering on the SP's Profit from CPs

In Fig. 4, PP incurs a loss when access reliability factor $\alpha$ is low in LAA, but when $\alpha$ is greater than a threshold, the SP's profit is always positive. On the contrary, when NN rules, the SP suffers from great losses. The simulation result is very close to that of the numerical simulation. Studies pointed out that SPs shift part of the spectrum cost to EUs, which avoid them from incurring losses [14]. We leave the question open for future research. When $c_{u}$ is 0.5 , it brings in greater losses for the SP. The decision for bandwidth is mainly based on costs; thus, lower costs will induce the SP to fetch more bandwidth, further devastating its profit. 
TABLE V. THE SP'S AND CP $i$ 's EQUILIBRIUM BEHAVIORS

\begin{tabular}{|l|l|l|}
\hline Optimal Unlicensed Amount $B_{u}{ }^{*}$ & $B_{u}{ }^{*} \in\left[G e^{-\left(2+c_{l}\right)}, G e^{-2}\right]$, solution to Eq. (14) \\
\hline Access Realization Factor $\alpha$ & $0 \leq \alpha \leq G e^{-\left(2+c_{l}\right)} / B_{u}{ }^{*}$ & $\alpha>G e^{-\left(2+c_{l}\right)} / B_{u}{ }^{*}$ \\
\hline Optimal Licensed Amount $B_{l}$ & $G e^{-\left(2+c_{l}\right)}-B_{u} \alpha$ & 0 \\
\hline Optimal Pricing $b_{0}^{*}$ & $1+c_{l}$ & $\ln \left(\frac{G}{B_{u}{ }^{*} \alpha}\right)-1$ \\
\hline Expected Profit from CPs $\pi^{C P}$ & $\pi^{C P}$ in Eq. (15) & $(1+\lambda) \frac{G}{B_{u}{ }^{*} \alpha}$ \\
\hline CP $i$ 's SNR & $(1+\lambda) e^{2+c_{l}}$ & $g_{i}(1+\lambda) \frac{B_{u}^{*} \alpha}{G}-b \lambda$ \\
\hline CP $i$ 's Payoff & $g_{i}(1+\lambda) e^{-\left(2+c_{l}\right)}-b \lambda$ \\
\hline
\end{tabular}

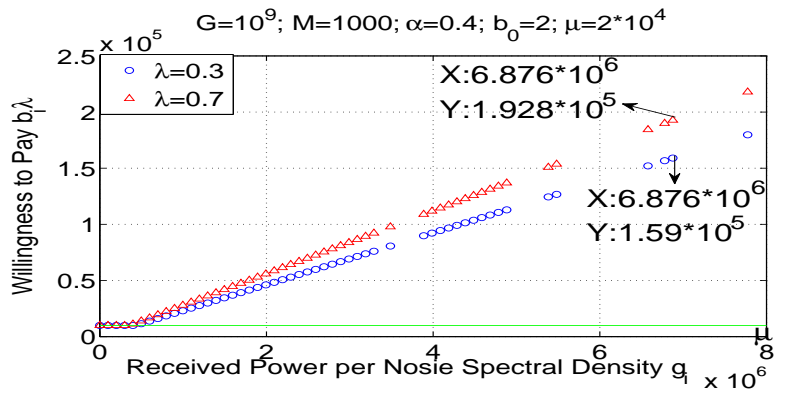

Fig. 5. Additional Fee $b \lambda$ and the Improvement on SNR $\lambda$

When PP exists, the $\mathrm{CPH}$ is granted premium access and contributes $b \lambda$ to the SP. In Fig. 5, given the same original received SNR $g_{i}$ (e.g., $4 \times 10^{6}$ ), when $\lambda=0.3$, the $b \lambda$ the $\mathrm{CPH}$ is willing to pay is around $9 \times 10^{4}$; when $\lambda$ increases to 0.7 , it pays a higher additional fee close to $1.2 \times 10^{5}$, reflecting a higher valuation towards greater improvement on SNR.

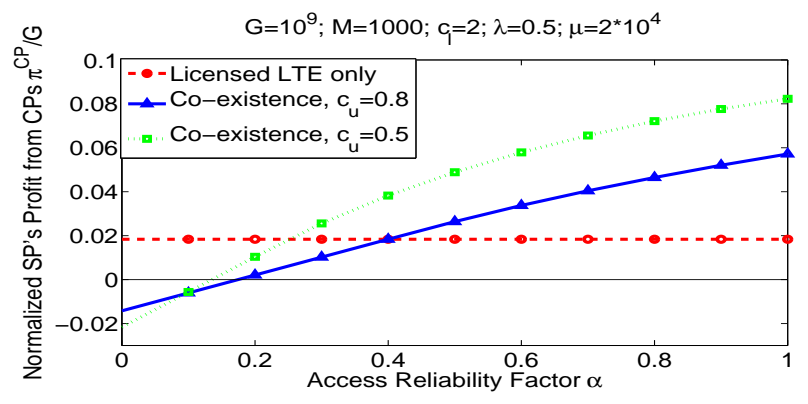

Fig. 6. The SP's normalized optimal realized profit from CPs as a function of $\alpha$

In Fig. 6, if the access reliability $\alpha$ in LAA is above a threshold, adopting LAA can bring extra normalized realized profit for the SP. The crossing feature of the two increasing curves is because the optimal unlicensed amount $B_{u}^{*}$ is larger under a lower cost of unlicensed bandwidth $\left(c_{u}=0.5\right)$, which leads to greater realized profit loss when $\alpha$ is close to zero. This shows the tradeoff between improvement on the expected profit and the large variability of the realized profit.

Proposition 3. CPs always benefit from the availability of unlicensed spectrum.

Proof 3. In the baseline approach without unlicensed bandwidth, the SP always charges the price $1+c_{l}$. As shown in Table $\mathrm{V}$, the equilibrium price $b_{0}^{*}$ with unlicensed bandwidth is always no larger than $1+c_{l}$ for any value of $\alpha$. Since CP $i$ 's payoff is strictly decreasing in price, CPs always benefit from the availability of unlicensed spectrum.

\section{CONCLUSiON}

We cope with a premium peering pricing model for content delivery in the co-existence of LTE and LAA wireless network. The pricing problem is solved with a four-stage Stackelberg game coupling with job market signaling game and second price auction. The result shows that the optimal amount of spectrum introduced by the SP is closely related to the access reliability factor in LAA. Moreover, the framework points out that on average CPs still benefit from premium peering access because of the increase in bandwidth and alleviation of excess demand. By properly subsidizing the SP, premium peering can generate appropriate revenues to both the SP and CPs.

\section{ACKNOWLEDGEMENT}

This work was in part supported by Ministry of Science and Technology under Grants MOST103-2221-E-002-086-MY.

\section{REFERENCES}

[1] METIS official website. Dec. 9, 2014. Retrieved from https://www.metis2020.com/, 2014.

[2] TR-36.889 V0.1.1. Study on licensed-assisted access to unlicensed spectrum. 3GPP Release 13, 2014.

[3] F.N. Abinader Jr. et al. Enabling the coexistence of LTE and Wi-Fi in unlicensed bands. IEEE Commun. Mag., 52(11):54-61, 2014.

[4] A. Medeisis, J. Sydor, L.C. Cremene, O. Holland, A. Anskaitis, D. Wiecek, Y. Haddad, and T. Cuzanauskas. ISM-Advanced: improved access rules for unlicensed spectrum. In 2014 IEEE DYSPAN, pages 194-205, 2014.

[5] L. Duan, J. Huang, and B. Shou. Investment and pricing with spectrum uncertainty: a cognitive operator's perspective. IEEE Trans. Mob. Comput., 10(11):1590-1604, 2011.

[6] M. Andrews, U. Özen, M. I. Reiman, and Q. Wang. Economic models of sponsored content in wireless networks with uncertain demand. In IEEE INFOCOM WKSHPS 2013, pages 345-350, 2013.

[7] P Hande, Mung Chiang, R Calderbank, and S Rangan. Network pricing and rate allocation with content provider participation. In IEEE INFOCOM 2009, pages 990-998, 2009.

[8] L. Gyarmati, N. Laoutaris, K. Sdrolias, and C. Rodriguez, P. Courcoubetis. From advertising profits to bandwidth prices. Dec.29, 2014. Retrieved from http://arxiv.org/pdf/1404.4208v3.pdf, 2014.

[9] W. Choi, S. Han, S. H. Jung, and T. Kang. Managerial operating ability and the association between accruals and future cash flows. In 2014 Am. Account. Assoc. Annu. Meet., 2014.

[10] M. Spence. Job market signaling. Q. J. Econ., 87(3):355-374, 1973.

[11] W. S. Vickrey. Counterspeculation, auctions, and competitive sealed tenders. J. Finance, 16(1):pp. 8-37, 1961.

[12] C. Prendergast. The provision of incentives in firms. J. Econ. Lit., 37(1):7-63, 1999.

[13] John G Riley and William F Samuelson. Optimal auctions. Am. Econ. Rev., 71(3):pp. 381-392, 1981.

[14] S. Sen, C. Joe-Wong, S. Ha, and M. Chiang. A survey of smart data pricing: past proposals, current plans, and future trends. ACM Comput. Surv., 46(2):1:1-1:37, 2013. 